\title{
Cerebral perfusion and glucose metabolism in Alzheimer's disease and frontotemporal dementia: two sides of the same coin?
}

\author{
Sander C. J. Verfaillie • Sofie M. Adriaanse • Maja A. A. Binnewijzend • \\ Marije R. Benedictus • Rik Ossenkoppele • Mike P. Wattjes • Yolande A. L. Pijnenburg • \\ Wiesje M. van der Flier • Adriaan A. Lammertsma • Joost P. A. Kuijer • \\ Ronald Boellaard • Philip Scheltens • Bart N. M. van Berckel • Frederik Barkhof
}

Received: 15 December 2014 / Revised: 19 February 2015 / Accepted: 24 February 2015 / Published online: 22 April 2015

(C) The Author(s) 2015. This article is published with open access at Springerlink.com

\begin{abstract}
Objectives Alzheimer's disease (AD) and frontotemporal (FTD) dementia can be differentiated using $\left[{ }^{18} \mathrm{~F}\right]$-2-deoxy-2fluoro-D-glucose (FDG)-PET. Since cerebral blood flow $(\mathrm{CBF})$ is related to glucose metabolism, our aim was to investigate the extent of overlap of abnormalities between AD and FTD.
\end{abstract}

All authors are affiliated with the Neuroscience Campus Amsterdam \& VU University Medical Centre

Electronic supplementary material The online version of this article (doi:10.1007/s00330-015-3696-1) contains supplementary material, which is available to authorized users.

S. C. J. Verfaillie $(\triangle) \cdot S$. M. Adriaanse $\cdot$ M. A. A. Binnewijzend

M. R. Benedictus $\cdot$ R. Ossenkoppele $\cdot$ M. P. Wattjes $\cdot$

A. A. Lammertsma - R. Boellaard - B. N. M. van Berckel •

F. Barkhof

Department of Radiology \& Nuclear Medicine, VU University

Medical Centre, Amsterdam, The Netherlands

e-mail: s.verfaillie@vumc.nl

S. M. Adriaanse

e-mail: s.adriaanse@vumc.nl

M. A. A. Binnewijzend

e-mail: M.Binnewijzend@ggzingeest.nl

M. R. Benedictus

e-mail: m.benedictus@vumc.nl

R. Ossenkoppele

e-mail: r.ossenkoppele@vumc.nl

M. P. Wattjes

e-mail: m.wattjes@vumc.nl

A. A. Lammertsma

e-mail: aa.lammertsma@vumc.nl

R. Boellaard

e-mail: r.boellaard@vumc.nl

B. N. M. van Berckel

e-mail: b.berckel@vumc.nl
Methods Normalized FDG-PET and arterial spin labelling (ASL-MRI)-derived CBF was measured in $18 \mathrm{AD}$ patients (age, 64 \pm 8 ), 12 FTD patients (age, 61 \pm 8 ), and 10 controls (age, 56 \pm 10 ). Voxel-wise comparisons, region-of-interest (ROI), correlation, and ROC curve analyses were performed. Results Voxel-wise comparisons showed decreased CBF and FDG uptake in AD compared with controls and FTD in both

F. Barkhof
e-mail: f.barkhof@vumc.nl

S. C. J. Verfaillie - S. M. Adriaanse $\cdot$ M. A. A. Binnewijzend M. R. Benedictus $\cdot$ R. Ossenkoppele $\cdot$ Y. A. L. Pijnenburg •

W. M. van der Flier $\cdot$ P. Scheltens

Alzheimer Centre and Department of Neurology, VU University Medical Centre, P.O. Box 7057, 1007

MB Amsterdam, The Netherlands

Y. A. L. Pijnenburg

e-mail: y.pijnenburg@vumc.nl

W. M. van der Flier

e-mail:wm.vdflier@vumc.nl

P. Scheltens

e-mail: p.scheltens@vumc.nl

W. M. van der Flier

Department of Epidemiology \& Biostatistics, VU University

Medical Centre, Amsterdam, The Netherlands

J. P. A. Kuijer

Department of Physics and Medical Technology, VU University

Medical Centre, Amsterdam, The Netherlands

e-mail: jpa.kuijer@vumc.nl 
precuneus and inferior parietal lobule (IPL). Compared with controls and AD, FTD patients showed both hypometabolism and hypoperfusion in medial prefrontal cortex (mPFC). ASL and FDG were related in precuneus $(r=0.62, p<0.001)$, IPL $(r=0.61, p<0.001)$, and mPFC across groups $(r=0.74$, $p<001)$. ROC analyses indicated comparable performance of perfusion and metabolism in the precuneus (AUC, 0.72 and 0.74$)$, IPL ( 0.85 and 0.94 ) for $\mathrm{AD}$ relative to FTD, and in the $\mathrm{mPFC}$ in FTD relative to AD (both 0.68).

Conclusions Similar patterns of hypoperfusion and hypometabolism were observed in regions typically associated with AD and FTD, suggesting that ASL-MRI provides information comparable to FDG-PET.

Key Points

- Similar patterns of hypoperfusion and hypometabolism were observed in patients with dementia.

- For both imaging modalities, parietal abnormalities were found in Alzheimer's disease.

- For both imaging modalities, prefrontal abnormalities were found in frontotemporal dementia.

Keywords Perfusion MRI · Positron-emission tomography · ${ }^{18}$ F-FDG $\cdot$ Alzheimer's disease $\cdot$ Frontotemporal dementia

\section{Introduction}

Alzheimer's disease (AD) and frontotemporal dementia (FTD) are among the most common types of dementia, and both are characterized by progressive impairment of cognitive, behavioural, and daily functioning $[1,2]$. Early in the course of the disease in particular, clinical presentation of FTD and AD may overlap or may be misdiagnosed as another psychiatric or neurological disorder. Brain glucose metabolism measured using $\left[{ }^{18} \mathrm{~F}\right]$-2-deoxy-2-fluoro-D-glucose $\left({ }^{18} \mathrm{~F}\right.$ FDG) positron emission tomography (PET) provides important additional diagnostic information about brain function [3]. Glucose metabolism reflects neuronal (dys)function, which is thought to be an early marker of dementia, preceding structural magnetic resonance imaging (MRI) findings such as cortical atrophy and clinical symptoms [4]. Bilateral temporal and parietal glucose hypometabolism is present in patients with AD of mild severity [5-7]. In contrast, patients with FTD show marked hypometabolism in the prefrontal cortex (PFC) [8-10]. For this reason, FDG is able to accurately differentiate between AD and FTD $[3,7,11]$ with a high level of specificity $[12,13]$.

The disadvantages of PET include radiation exposure and the relatively high cost. A potential alternative to FDG-PET is the assessment of cerebral blood flow (CBF) measured by perfusion MRI, as $\mathrm{CBF}$ is thought to be closely related to metabolism [14]. CBF can be measured non-invasively using arterial spin labelling (ASL), which makes use of magnetically labelled water in the arterial blood supply as an endogenous tracer $[15,16]$. Indeed, comparisons between CBF measured with ASL and single-photon emission computed tomography provided almost equivalent diagnostic information in $\mathrm{AD}$ [17]. As such, like FDG, ASL may be suitable to identify unique disease-related patterns.

Among the advantages of ASL are its relatively short acquisition time, low cost, lack of radiation exposure, non-invasiveness, and easy incorporation into standard MRI protocols. ASL studies in patients with AD have shown lower CBF in parietal and temporal regions and in the posterior cingulate cortex (PCC) [18-21], very similar to findings with FDG. In FTD, lower levels of CBF were found in the right frontal regions in comparison to controls, while higher $\mathrm{CBF}$ levels were found in the temporal-parietal regions and PCC compared to $\mathrm{AD}[22,23]$.

The first study directly comparing FDG and ASL between patients with $\mathrm{AD}$ and healthy subjects showed corresponding deficits primarily in the angular gyrus and PCC [24, 25]. As such, ASL may have potential clinical use in the diagnostic workup of dementia. While ASL sequences could be available in nearly every hospital equipped with MRI, perfusion maps are not yet commonly used for diagnostic decision-making. ASL is also a relatively new technique and needs further validation against the more established FDG-PET. Before ASL can serve as a more solid biomarker for differential diagnosis in single subjects, several dementia types must first be compared at a group level with ASL compared to FDG imaging. To date, no study has compared ASL with FDG in FTD, or compared FTD with AD together with its discriminative performance. The use of a retrospective design allowed us to investigate brain function in patients with a mild disease state while ensuring that a clinical diagnosis was maintained at approximately two years. Therefore, the aim of this study was to compare FDG (metabolism) and ASL (perfusion) imaging in the same subjects, and more importantly, to determine the extent of overlap with regard to aberrant brain function between the imaging techniques.

\section{Methods}

Subjects

A convenience sample comprising 18 patients with $\mathrm{AD}, 12$ patients with behavioural-variant FTD, and 10 controls with available FDG and ASL images from the Amsterdam Dementia Cohort were included [26]. Subjects were eligible if the interval between FDG and ASL examinations was 6 months or less. All patients visited the VU University Medical Centre outpatient memory clinic between October 2010 and October 
2012. All underwent standardized dementia screening: medical history, neuropsychological assessment, physical and neurological examinations, laboratory testing, and a structural brain MRI. Clinical diagnosis was established by consensus among a multidisciplinary team prior to PET imaging, and without knowledge of the ASL data.

AD patients fulfilled the National Institute of Neurological and Communicative Disorders and Stroke/Alzheimer's Disease and Related Disorders Association criteria for probable $\mathrm{AD}$ [27]. Alzheimer's pathology was verified in all AD patients using non-displaceable binding potential parametric images based on 90-min dynamic Pittsburgh Compound-B (PIB) PET scans. All FTD patients met the criteria for probable behavioural-variant FTD [28]. AD and FTD patients were eligible if the diagnosis was maintained over a clinical follow-up period of at least 1 year. Controls were patients with subjective memory complaints who lacked verified cognitive disorders or any relevant psychiatric disorders, and who showed no evidence of neurodegenerative disorder on MRI [19]. In addition, controls were required to have normal CSF Aß1-42 levels in order to exclude possible preclinical AD cases.

The study was approved by the Medical Ethics Review Committee, and all subjects provided written informed consent.

\section{PET}

Fifteen minutes prior to injection, patients were instructed to rest with their eyes closed and wearing earplugs in a dimly lit room with minimal background noise. Forty-five minutes after intravenous bolus injection of $188 \pm 8 \mathrm{MBq}{ }^{18} \mathrm{~F}-\mathrm{FDG}$, patients underwent a 15 -min emission examination $(3 \times 5$-min frames). FDG emission scans were obtained using a Gemini TF 64 PET-CT (Philips Medical Systems, Cleveland, OH, USA) or ECAT EXACT HR+ (Siemens/CTI, Knoxville, TN, USA) scanner (AD, $\mathrm{n}=6 / \mathrm{n}=12 ; \mathrm{FTD}, \mathrm{n}=7 / \mathrm{n}=5$; controls $n=3 / n=7$, respectively). Details with respect to image acquisition have been described elsewhere [29]. All PET unreconstructed data (sinograms) were normalized and corrected for random events, dead time, scatter, and decay. Attenuation correction was performed using a low-dose CT (PET-CT) or transmission scan (ECAT HR+). The reconstruction protocol has been described elsewhere [30], but included standard reconstruction algorithms for both systems (ECAT HR+: standard filtered back-projection; PET-CT 3D row-action maximum likelihood algorithm). Finally, standardized uptake values (SUVs) were calculated, normalized for injected dose and body weight and length, using an in-house-developed software tool for kinetic and parametric analysis of dynamic PET studies. SUV images were normalized to global uptake using SPM8 (Statistical Parametric Mapping; Wellcome Trust Centre for Neuroimaging, London, UK).
MRI

MR images were acquired on a $3 \mathrm{~T}$ whole-body MR system (Signa HDxt; GE Medical Systems, Milwaukee, WI, USA) using an 8-channel head coil. The MRI acquisition protocol has been extensively described elsewhere [19], and included a sagittal 3D T1-weighted inversion recovery fast spoiled gradient echo. ASL-MRI sequencing was provided by GE. Pseudo-continuous ASL perfusion images (3D fast spinecho acquisition with background suppression; post-label delay, $2.0 \mathrm{~s}$; repetition time $[\mathrm{TR}]=9 \mathrm{~ms}$; echo time $[\mathrm{TE}]=4.8 \mathrm{~s}$; spiral readout, 8 arms $\times 512$ samples; $36 \times 5.0 \mathrm{~mm}$ axial slices; $3.2 \times 3.2 \mathrm{~mm}^{2}$ in-plane resolution; reconstructed pixel size, $1.7 \times 1.7 \mathrm{~mm}^{2}$; acquisition time, $4 \mathrm{~min}$ ) were calculated after subtracting labelled from control images. Both T1-weighted and ASL images were corrected for gradient non-linearity in all three directions. Perfusion was normalized to global perfusion using SPM8, as described for FDG.

Image analysis

Pre-processing and between-group image comparisons were performed using SPM8 in MATLAB (MathWorks, Release 2010a). Parametric images of FDG-SUV and ASL-derived $\mathrm{CBF}$ were co-registered to the structural $\mathrm{T} 1 \mathrm{image}$, and spatially registered to the Montreal Neurological Institute (MNI) space (voxel size, $2.0 \times 2.0 \times 2.0 \mathrm{~mm}^{3}$ ) [31]. ASL images were spatially smoothed using an 8 -mm full-width-at-halfmaximum Gaussian kernel corresponding to the smoothing kernel of FDG images at reconstruction. Group effects were investigated separately for ASL and FDG with a voxel-wise contrast including covariates (age and sex) to adjust for common confounding effects [32]. To reliably and validly compare normalized signals of the techniques in grey matter, proportional scaling, mean global calculation, and grey matter masking was applied in SPM8. Because we were interested in the concordance of spatial abnormality patterns and corresponding standard normal deviate relative to controls, SPMderived mean z-scores were extracted from multiple significant clusters (thresholded at $p<0.001$, uncorrected for multiple comparisons [cluster size $\geq 10]$ ).

To investigate the extent to which FDG and ASL were correlated, region-of-interest (ROI) analyses were performed. Based on the most significant clusters derived from patientversus-control comparisons that (partially) overlapped between FDG and ASL, post hoc ROI analysis was performed using MarsBaR (MARSeille Boîte À Région d'Intérêt [Marseille Region of Interest Toolbox]) (v 0.43, Marseille, France). Functional ROIs encompassed both FDG and ASL regional abnormal clusters at a liberal threshold (defined through SPM local maxima $p<0.05$ uncorrected), ensuring a target region of adequate size. 
In total, three separate ROIs were created for $\mathrm{AD}(n=2)$ and FTD $(n=1)$ signature regions (Supplementary Fig. 2). ROI coordinates for $\mathrm{AD}$ were as follows: precuneus (xyz ASL $-10 /-68 / 30$, FDG $-2 /-70 / 32$ ) and inferior parietal lobule (IPL) (xyz ASL 44/-56/46, FDG 46/-58/40). ROI coordinates for FTD were mPFC (xyz ASL 2/62/6, FDG 6/60/6). Next, a combined (ASL+FDG) signature ROI was saved as a single cluster, and trimmed with a box $\left(20 \times 20 \times 20 \mathrm{~mm}^{3}\right)$ at intermediate voxel coordinates (precuneus: $-6 /-69 / 31$, IPL: 45/-57/43, mPFC: 4/61/6). Trimming was done to remove extraneous voxels (i.e. outside signature region). ROI values were extracted with default settings.

\section{Statistical analysis}

Demographic and clinical data were analysed using SPSS software (IBM SPSS Statistics for Windows Version 20.0; IBM Corp., Armonk, NY, USA). If data deviated from normality (using Kolmogorov-Smirnov tests for non-normality), non-parametric tests were used, as indicated. Demographic data (age, weight, and body length) and MMSE were assessed using analysis of variance (ANOVA). Gender differences were assessed using $\chi^{2}$ tests. Across groups, the relationship between FDG and ASL was investigated using the Pearson correlation.

Across-group (AD, FTD, controls) comparative performance was investigated using binary logistic regression, with all FDG and ASL ROI values entered simultaneously as input, and a dummy-coded dementia group as dependent variable. Multicollinearity assumptions were investigated using linear regression methods. Further, to investigate the extent to which FDG and ASL were able to discriminate between AD and FTD, receiver operating characteristic (ROC) curves were generated to obtain an area under the curve (AUC). Specifically, separate ASL and FDG ROI values from AD and FTD patients were used as input (test variable); the dementia group was set as state variable. In addition, mean difference scores of signature ROIs were calculated to determine whether subtracting signature regions would result in better discriminative performance.

\section{Results}

Demographic and clinical variables are presented in Table 1. Age, gender, time intervals, and FDG SUV normalization variables (body weight and length, injected dose) did not differ among groups. Likewise, there was no difference in frequency distribution of PET systems (PET-CT and HR+) among the groups $\left(\chi^{2}(2)=1.6, p=0.21\right)$. The combined dementia groups showed lower MMSE scores $(\mathrm{F}[38,1])=1.8$, $p=0.01$ ) than controls, but did not differ between each other. All amyloid PET scans of the AD patient group were classified as consistent with abnormal amyloid load. All FTD patients had normal cerebrospinal fluid (CSF) Aß1-42 levels $(n=3)$ or negative amyloid PET scan $(n=8)$. In one FTD patient, amyloid-specific information was missing. Figure 1 shows raw ASL and FDG images with corresponding MRI of a typical AD and FTD patient.

Figure 2 shows regional abnormalities derived from group comparisons projected onto an MNI glass brain, while Fig. 3A shows z-scores for FTD and AD compared to controls (MNI coordinates with corresponding cluster sizes are displayed in Supplementary Table 2). In AD compared to controls, both lower metabolism (FDG) and lower perfusion (ASL) were found in the bilateral precuneus, bilateral inferior parietal lobule (IPL), and dorsolateral prefrontal cortex (DLPFC). In addition, AD patients showed lower perfusion in the orbitofrontal cortex (OFC). Compared with controls, FTD patients showed both lower metabolism and lower perfusion in $\mathrm{mPFC}$, OFC, and temporal poles. In addition, hypoperfusion was found in the supplementary motor area (SMA) and hypometabolism in the DLPFC in FTD. Overall, mean ageand sex-adjusted z-scores (reflecting normal deviates) were comparable between ASL and FDG in patient groups relative to controls.

Compared with FTD patients, patients with AD showed lower metabolism in the precuneus and bilateral IPL, while lower perfusion was observed in the precuneus and left IPL (Fig. 3B). In addition, lower perfusion was found in the middle temporal gyrus (MTG) with AD compared to FTD, with the opposite pattern in the SMA, OFC, temporal poles, and mPFC. Likewise, lower metabolism was found in the OFC, SMA, mPFC, temporal poles, and anterior PFC (aPFC) in FTD relative to AD. While OFC hypometabolism was located in anteromedial orbitofrontal regions, hypoperfusion was found predominantly in posterolateral regions, extending to the temporal poles.

Correlations between regional FDG and ASL values are shown in Fig. 4. Across groups, a strong correlation between FDG and ASL was found in mPFC $(r=.74, p<0.001$; Fig. 4A), IPL ( $r=.61, p<0.001$; Fig. 4B), and precuneus $(r=.62, p<0.001)$.

The predictive value of the separate ROIs ([IPL, precuneus, $\mathrm{mPFC}] \times \mathrm{FDG}+\mathrm{ASL})$ in all groups $(\mathrm{AD}, \mathrm{FTD}$, controls) for $\mathrm{AD}$ and FTD was investigated using logistic regression. There was a significant fit of the data in $\operatorname{AD}\left(\chi^{2}(6)=44.1, p<0.001\right)$ predicted by IPL metabolism ( $\beta \pm \mathrm{SE}, 0.54 \pm 0.31, p=0.046$ ), but not by IPL $(\beta \pm \mathrm{SE}, 0.71 \pm 0.31)$ or precuneus $(\beta \pm \mathrm{SE}, 0.88$ \pm 0.12 ) perfusion, or precuneus ( $\beta \pm \mathrm{SE}, 0.94 \pm 0.1113$ ) metabolism. Testing was performed to exclude significant multicollinearity, and showed no violations (all variables: tolerance $>0.41, \mathrm{VIF}<2.46$ ). In contrast, for FTD, there was a significant fit of the data $\left(\chi^{2}(6)=32.1, p<0.001\right)$ predicted by $\mathrm{mPFC}$ perfusion $(\beta \pm \mathrm{SE}, 0.74 \pm 0.16, p=0.026)$, but not mPFC $(\beta \pm$ SE, $0.89 \pm 0.15)$ metabolism. Multicollinearity 
Table 1 Demographic and clinical data

\begin{tabular}{|c|c|c|c|c|}
\hline $\begin{array}{l}\text { Patient group } \\
\text { Characteristics }\end{array}$ & $\begin{array}{l}\mathrm{AD} \\
N=18\end{array}$ & $\begin{array}{l}\text { FTD } \\
N=12\end{array}$ & $\begin{array}{l}\text { Controls } \\
N=10\end{array}$ & Test-statistic ( $p$ value) \\
\hline Age in years (SD) & $64(8)$ & $61(8)$ & $56(10)$ & $\mathrm{F}(37,2)=1.9, p=0.17$ \\
\hline Gender & $61 \%$ male & $42 \%$ male & $90 \%$ male & $\chi 2(2)=5.5, p=0.06$ \\
\hline MMSE (SD) & $24(4)$ & $24(4)$ & $27(3)$ & $\mathrm{F}(37,2)=2.6, p=0.09$ \\
\hline Median years of complaints (interquartile range)* & $2.0(1.9)$ & $2.3(1.9)$ & n.a. & $\mathrm{U}=101.5, p=0.78$ \\
\hline Scan interval in months (SD) & $2.1(1)$ & $2.2(2)$ & $1.9(2)$ & $\mathrm{F}(37,2)=.8, p=0.5$ \\
\hline \multicolumn{5}{|l|}{ FDG SUV normalization variables } \\
\hline \multicolumn{5}{|l|}{ Body } \\
\hline Weight in kg (SD) & $78(11)$ & $75(15)$ & $86(17)$ & $\mathrm{F}(37,2)=2.2, p=0.13$ \\
\hline Length in cm (SD) & $176(9)$ & $169(10)$ & $175(9)$ & $\mathrm{F}(37,2)=1.5, p=0.23$ \\
\hline Injected dose in $\mathrm{MBq}(\mathrm{SD})$ & $190(8)$ & $187(8)$ & $188(7)$ & $\mathrm{F}(37,2)=.97, p=0.39$ \\
\hline
\end{tabular}

Abbreviations: $\mathrm{cm}$ centimetre, $\mathrm{kg}$ kilogram, $M B q$ megabecquerel, $S D$ standard deviation, $S U V$ standardized uptake value

* Obtained through interviews with relatives of patients

testing showed no violations (all variables: tolerance $>0.40$, $\mathrm{VIF}<2.52$ ).

To investigate the discriminative power of ASL and FDG for $\mathrm{AD}$ and FTD, ROC curves were generated. Figure 5 shows

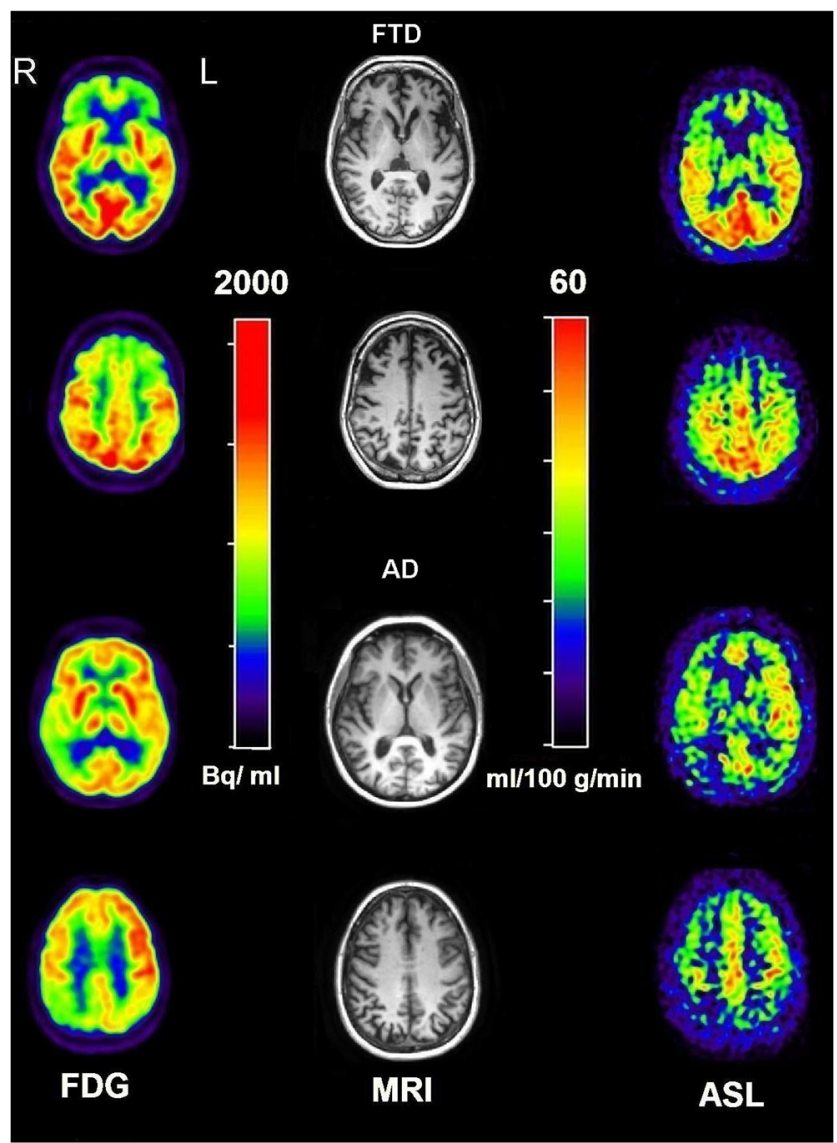

Fig. 1 Transversal FDG and ASL images of an FTD (first and second rows, MMSE 26) and an AD (third and fourth rows, MMSE 17) patient with early-onset disease. Both transversal planes show predominantly prefrontal abnormalities in FTD and parietal abnormalities in AD. Red colour reflects normal metabolism and perfusion
ROC curves for $\mathrm{AD}$ and FTD based on precuneus (Fig. 5A) and $\mathrm{mPFC}$ (Fig. 5B) ROI values. Precuneus AUC was 0.74 for FDG and 0.72 for ASL. For IPL, the AUC was 0.94 for FDG and 0.85 for ASL. For mPFC, the AUC was 0.68 for both FDG and ASL. Additionally, ratios (between $\mathrm{mPFC}$ and precuneus, and $\mathrm{mPFC}$ and IPL) were used for discriminating AD from FTD, which showed AUC values of 0.80 and 0.90 for the precuneus, and 0.89 and 0.96 for the IPL for FDG and ASL, respectively. Likewise, for discriminating FTD from AD, mPFC showed an AUC of 0.82 for FDG and 0.89 for ASL.

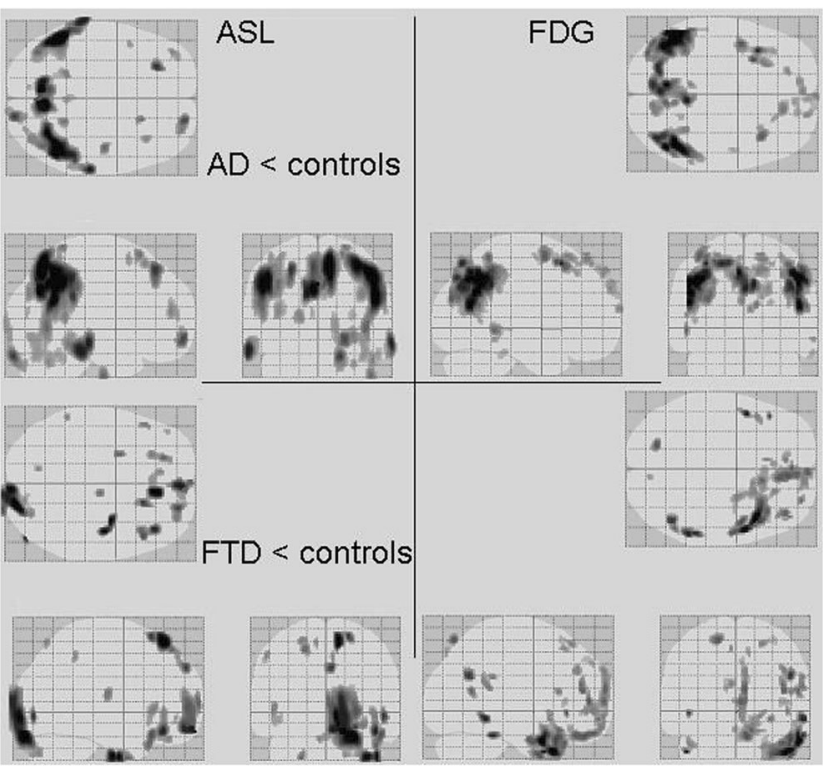

Fig. 2 Functional brain abnormalities of AD and FTD compared to controls projected onto a MNI glass brain. Predominantly parietal, precuneus aberrant function is visible in $\mathrm{AD}$ compared to controls, while FTD compared to controls shows mostly prefrontal abnormalities with both FDG and ASL. For illustrative purposes, images were thresholded at $p<0.005$ 
Fig. 3 Panel $A$ shows FDG and ASL regional mean z-scores of $\mathrm{AD}$ (left) and FTD (right) compared to controls. Panel B shows $\mathrm{AD}<\mathrm{FTD}($ left $)$ and FTD $<\mathrm{AD}($ right) perfusion and metabolism abnormalities

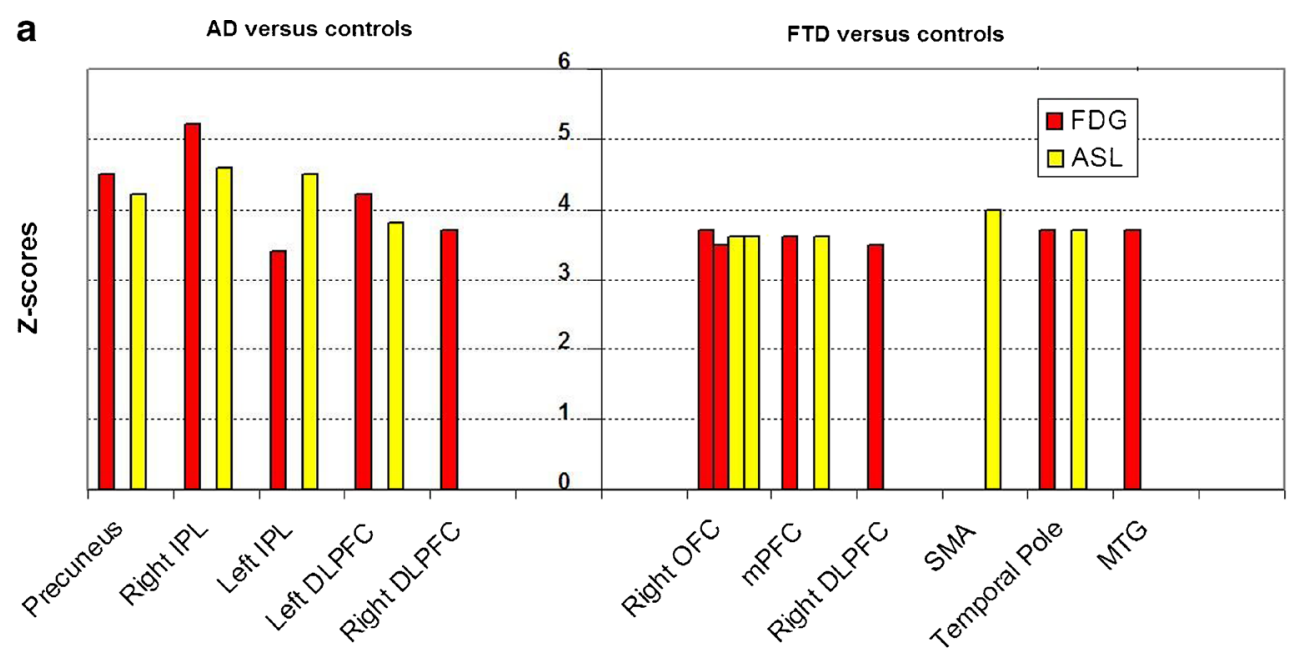

Brain Regions

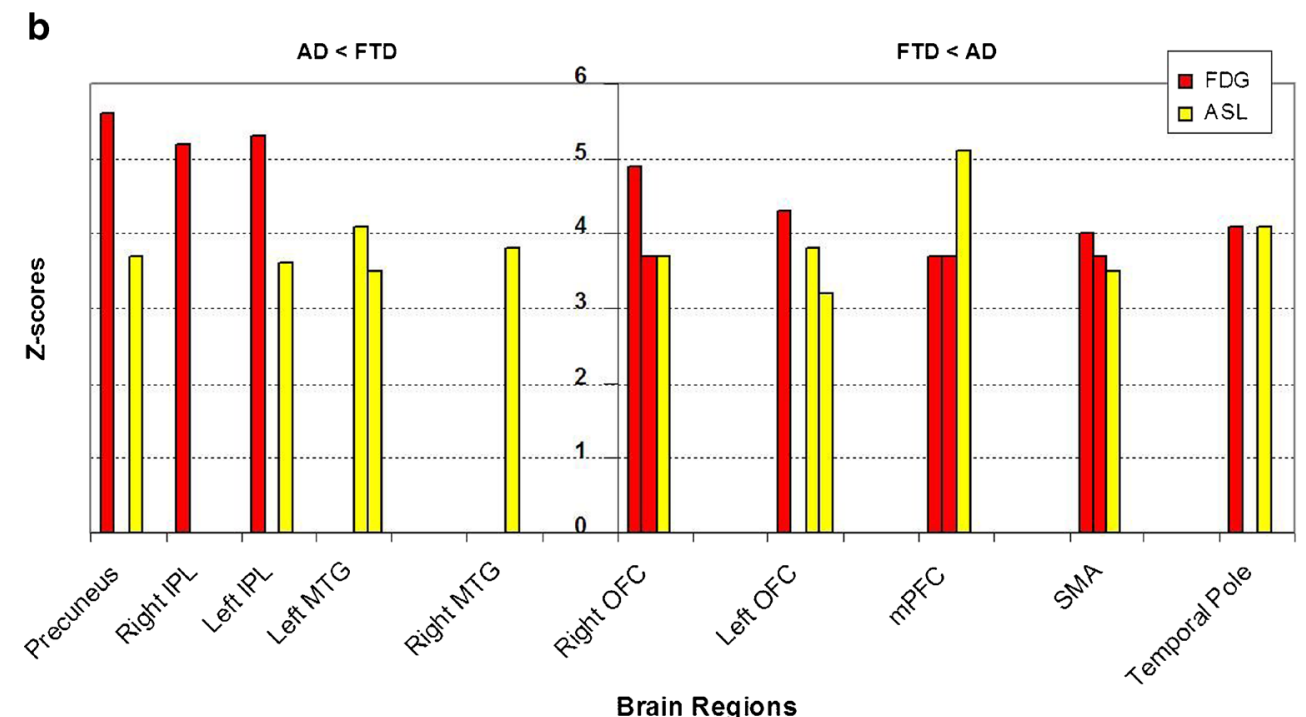

\section{Discussion}

In this study, FDG and ASL were compared between AD and FTD patients. The main finding of this study was that similar spatial abnormalities in glucose metabolism and perfusion were seen in AD and FTD patients compared with cognitively normal subjects in areas consistent with disease models. Overall, these findings reinforce the role for ASL in assessing spatial patterns of aberrant brain function in dementia, and suggest the potential for use in memory clinics.

To date, quantitative and qualitative FDG studies in $\mathrm{AD}$ have consistently demonstrated glucose hypometabolism in the bilateral temporal-parietal and precuneal regions $\mathrm{AD}$ [5-7], which are associated with neuropsychological deficits [33] and structural MRI atrophy [34]. The present FDG results on $\mathrm{AD}$ are in line with previous reports, indicating severely abnormal metabolism, especially in bilateral IPL.
The present study also demonstrated ASL hypoperfusion in bilateral IPL in patients with AD compared with controls, consistent with a previous study using voxel-wise analyses [21]. In another study, unilateral IPL hypoperfusion was seen [20], possibly as a result of globally decreased perfusion [19]. Hypoperfusion was also observed in the bilateral precuneus in AD patients compared with controls. These results are all in line with voxel-wise ASL studies in AD reporting decreased $\mathrm{CBF}$ in the PCC and parietal cortex compared with controls [18-21].

With regard to FTD, metabolic impairment, often asymmetric, has been reported in the frontal, basal ganglia, anterior cingulate cortex, and temporal regions [7-9]. The present FDG results are partially in line with these existing reports, as hypometabolism was observed in the frontal lobes and temporal poles but not in basal ganglia. One possible explanation may relate to the fact that subcortical metabolic deficits are 
a

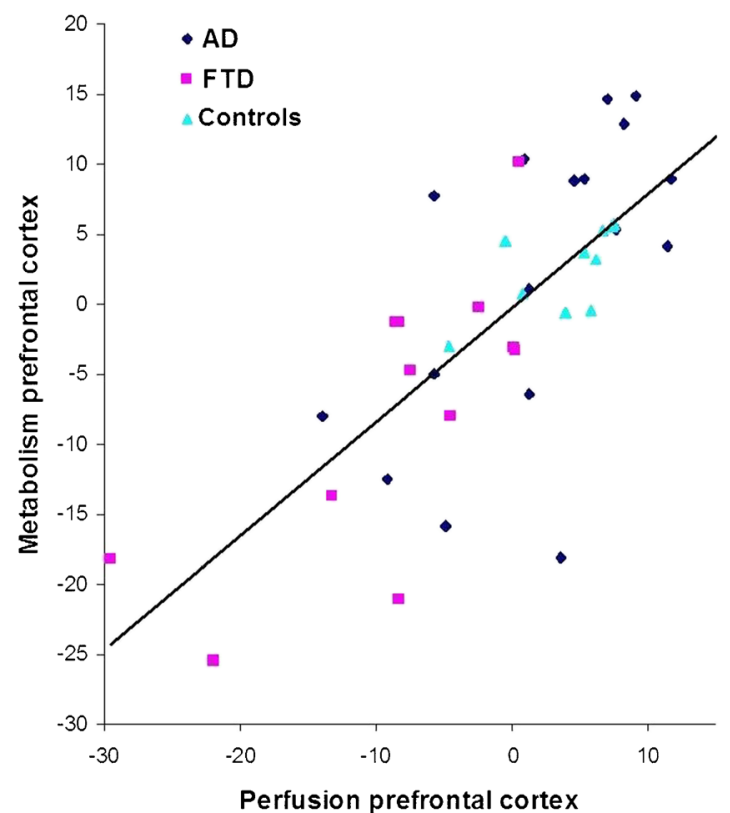

b

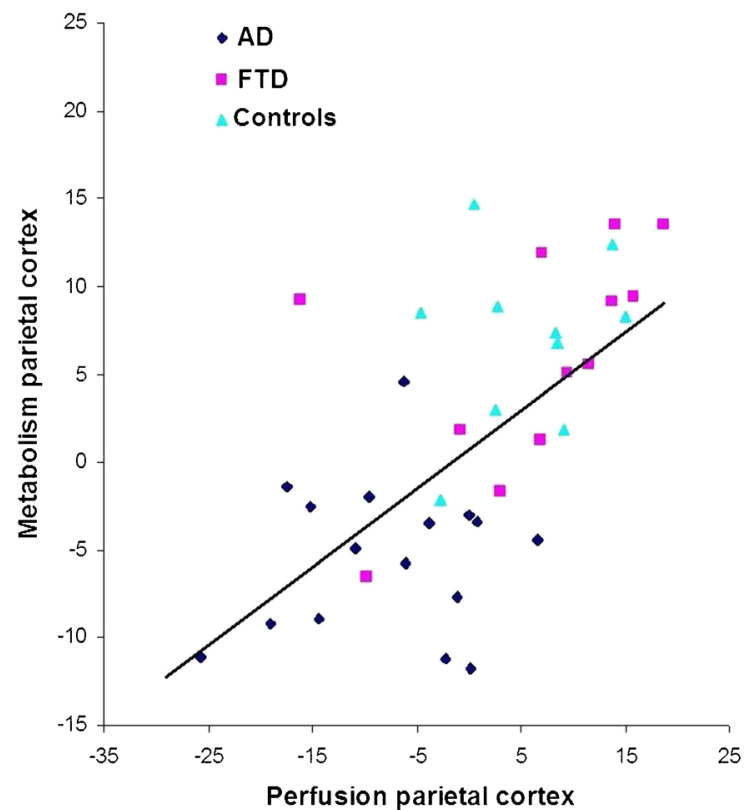

Fig. 4 Positive correlations between FDG SUV and ASL derived CBF in $(A)$ medial prefrontal cortex $(r i g h t, \mathrm{r}=.74, \mathrm{p}<0.001)$ and $(B)$ inferior parietal lobule (left, $r=.61, \mathrm{p}<0.001)$ across groups

associated with advanced disease states $[9,10]$ or comorbid motor neuron disease [8]. In the present study, however, behavioural-variant FTD patients who were included were primarily those with mild dementia.

In FTD compared to controls, functional abnormalities in the frontal lobe were found with ASL, consistent with previous studies [22, 23]. While some studies have demonstrated bilateral [23] and right [22] frontal deficits, the present findings predominantly point to dorsomedial prefrontal hypoperfusion. Previous neuroanatomical discrepancies may be explained by the finding that specific prefrontal functional abnormalities are associated with different behavioural FTD characteristics [35].

Overall, similar functional deficits were found in $\mathrm{AD}$ and FTD with both FDG and ASL, and these are consistent with well-known pathophysiological disease models [4, 28, 35]. The two imaging techniques showed comparable discriminative performance in terms of AUC. For AD signature ROIs, FDG showed slightly larger AUCs than ASL, whereas the opposite was found if we took the mean difference between a

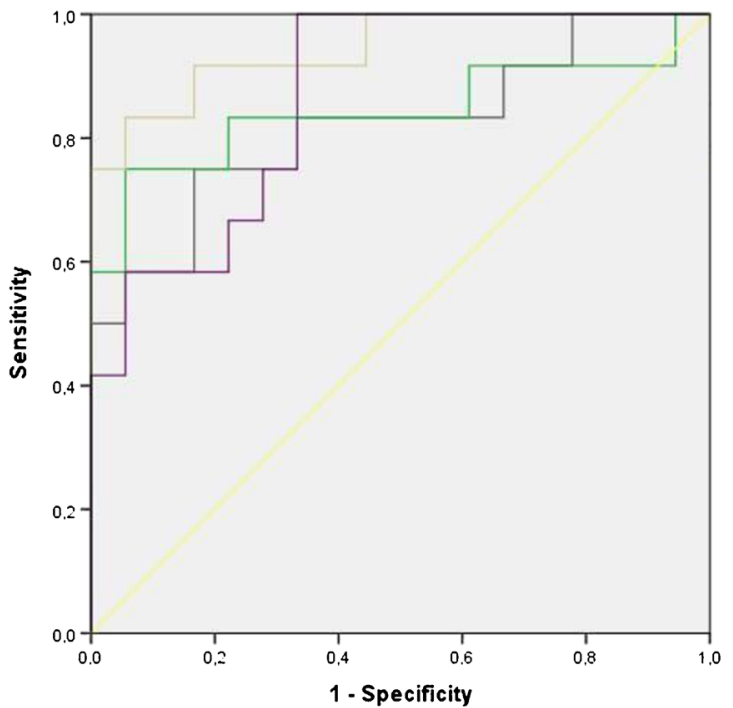

b

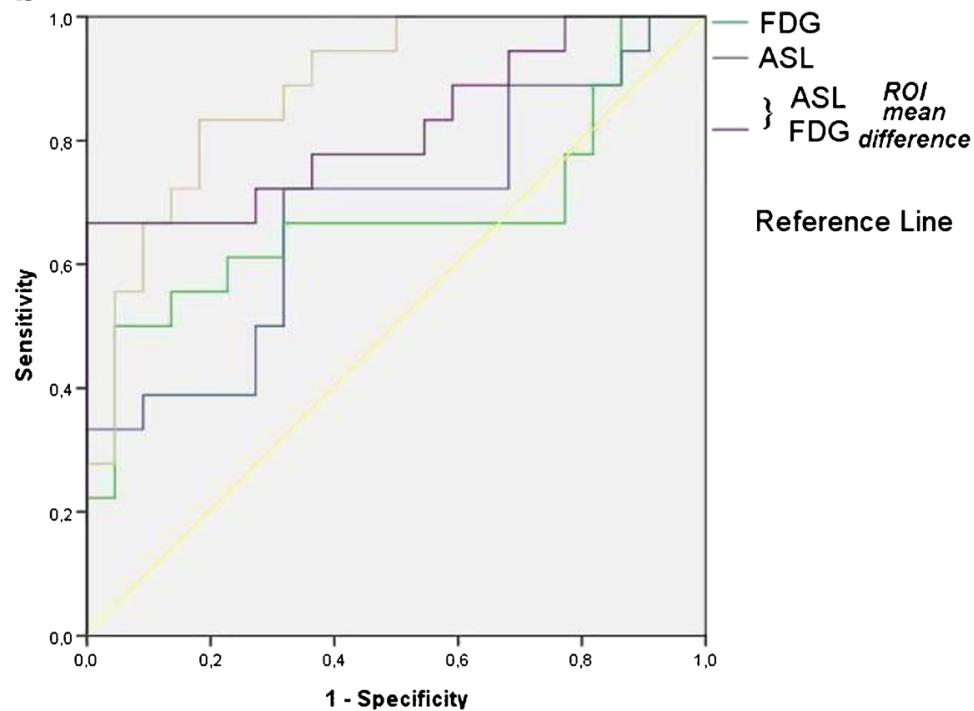

Fig. 5 ROC curves for FDG and ASL in AD and FTD. $A$ ) Precuneus function for ASL and FDG in discriminating AD from FTD. $B$ ) $m$ PFC function in discriminating FTD from AD. Ratios were taken between the $\mathrm{MPFC}$ and precuneus and entered into ROC analyses as 'ratio ROI' 
$\mathrm{AD}$ and FTD ROIs. For AD, the IPL showed slightly better differentiation from FTD compared to the precuneus with both FDG and ASL. In addition, regression analyses indicated that IPL metabolism was the strongest predictor for AD compared to all other ROIs, versus ASL prefrontal perfusion in FTD. Nevertheless, regional FDG and ASL values were correlated in the precuneus, IPL, and, most clearly, in the frontal lobe across groups. In contrast, voxel-wise abnormalities were most comparable in $\mathrm{AD}$ versus controls, and to a lesser extent in FTD. This might be caused by distinct and broad frontal deficiencies known to be present in FTD. To date, only one study has been reported in which a voxel-wise comparison between ASL and FDG was performed in AD. Chen et al. showed overlapping functional abnormalities in the PCC and angular gyri [24]. The present results endorse those findings, but additionally provide first evidence of largely overlapping functional abnormalities in imaging techniques for FTD already at an early stage with only mild disease severity. The current findings further demonstrate comparable discriminatory ability for FDG and ASL between the two most common early-onset dementia groups.

While overlapping ASL perfusion abnormalities relative to FDG were demonstrated at a group level, future studies are necessary to clarify the clinical potential of this application for single subjects, while also controlling for common confounding effects. The capabilities of ASL should also be investigated in more complex dementia cases or variants, or even in other neuropsychiatric disorders. In addition, to ensure general use, performance should be tested on different MRI systems. Lastly, for potential clinical use, visualization and assessment of CBF maps could benefit from scientific agreement on smoothing kernels for optimal signal-to-noise ratio.

Functional brain imaging may be hampered by partial volume effects (PVE) that can occur due to cerebral atrophy. Since our primary aim was to compare imaging technique performance in such a way that it could be extrapolated to a realistic clinical setting, our data was uncorrected for PVE. We therefore cannot distinguish true decreases in brain tissue perfusion and metabolism from apparent decreases in brain function due to atrophy. However, even though partial volume corrections (PVCs) were not performed CBF has been determined previously both with and without PVC across several dementia groups [36]. Binnewijzend and colleagues (2014) showed that after PVC, CBF consistently increased, but proportional differences remained similar across groups. Furthermore, there are concordant reports of functional brain abnormalities persisting after PVC in AD and FTD [37-39]. In addition, similar processing pipelines were used for FDG and ASL in the current study, and applying these corrections could have introduced unwanted variability in key brain regions. At this time, no ideal PVCs are available for either ASL or FDG [40].

This study had several other potential limitations. We used a convenience sample comprising patients with extensive differential diagnoses, which may have biased our results in that clear-cut cases (i.e. FDG scan not necessary) were not included. For this reason, not all FTD patients exhibited frontotemporal atrophy on MRI. Notwithstanding this issue, the study did show overlapping aberrant function already in cases with mild disease severity, which renders it suitable for early diagnosis. Another limitation was that subjects with cognitive complaints but without any verified abnormalities were used as a reference group, which consisted primarily of males. Despite statistically adjusting analyses for gender effects, this might have influenced our results. On the other hand, Liu and colleagues showed no specific regional hypoperfusion related to gender effects [32]. It is thus likely that our regional abnormalities do reflect dysfunction related to AD and FTD pathology. Furthermore, current knowledge is limited with regard to brain function in healthy subjects with cognitive complaints, although one could argue that more rigorously defined controls might be 'healthier' than the 'normal' elderly population, and that our control group better reflects clinical practice. Lastly, many uncontrolled factors such as smoking and caffeine intake may have affected measurements to a certain extent.

In conclusion, comparable reduced cerebral perfusion and metabolism were observed, predominantly in the parietal cortex and PCC in AD and in the frontal lobes in FTD, in line with pathophysiological disease models. As such, ASL can be considered as a promising adjunct to clinical investigations, even for early diagnosis.

Acknowledgments Research of the VUmc Alzheimer Center is part of the neurodegeneration research program of the Neuroscience Campus Amsterdam. The authors thank Ajit Shankaranarayanan of GE Healthcare for providing the $3 \mathrm{D}$ pseudo-continuous ASL sequence that was used to obtain data for this paper.

The scientific guarantor of this publication is Prof. Dr. Frederik Barkhof. The authors of this manuscript declare relationships with the following companies:

Dr. F. Barkhof serves on the editorial boards of Brain, European Radiology, Radiology, Multiple Sclerosis, Neuroradiology and Neurology, and serves as a consultant for Bayer Schering Pharma, Sanofi-Aventis, Biogen Idec, TEVA, Synthon BV, Merck Serono, Jansen Alzheimer Immunotherapy, Novartis, Genzyme, and Roche. Dr. Barkhof receives research support from the Dutch MS Society (EU-FP7). Dr. Barkhof has received consulting fees or honoraria for the consultancy mentioned above.

Dr. B.N.M. van Berckel receives research support from the Alzheimer Assistance Foundation, the Center for Translational Molecular Imaging, the Alzheimer Association, De Hersenstichting Nederland, and the Internationale Stichting Alzheimer Onderzoek. Dr. van Berckel receives no personal compensation from these organizations.

Dr. Wiesje van der Flier has received research funding and speaker honoraria from Boehringer Ingelheim; all funds were paid to her institution.

Dr. P. Scheltens receives grant support (for the institution; the VUmc Alzheimer Center, see also below) from GE Healthcare, Danone Research and Merck. In the past 2 years, he has received speakers' fees (paid to the institution) from Lilly, GE Healthcare, Lundbeck, Danone, and Janssen AI-Pfizer. 
Dr. Wattjes serves as a consulant for Biogen Idec and Roche and as an editorial board member of European Radiology.

S.C.J. Verfaillie, S.M. Adriaanse, M.A.A. Binnewijzend, M.R. Benedictus, R. Ossenkoppele, A.A. Lammertsma, Y.A.L. Pijnenburg, R. Boellaard, and J.P.A. Kuijer report no disclosures.

The authors of this manuscript declare no relationships with any companies whose products or services may be related to the subject matter of the article.

This study has received funding by Research of the VUmc Alzheimer Center. The VUmc Alzheimer Center is supported by Alzheimer Nederland and Stichting VUmc Fonds.

Dr. W.M. van der Flier and Dr. J.P.A. Kuijer (authors) kindly provided statistical advice for this manuscript. Institutional review board approval was obtained. Written informed consent was obtained from all subjects (patients) in this study. Some study subjects have been previously reported in Eur Radiol. 2014 Sep;24(9):2326-33. doi: 10.1007/s00330-0143172-3. Five of 12 ASL scans of FTD patients and five of 18 ASL scans of AD patients have been used before. None of the FDG and ASL control scans have been published previously. Methodology: retrospective crosssectional study, performed at one institution.

Open Access This article is distributed under the terms of the Creative Commons Attribution Noncommercial License which permits any noncommercial use, distribution, and reproduction in any medium, provided the original author(s) and the source are credited.

\section{References}

1. Alzheimer's Association (2012) 2012 Alzheimer's disease facts and figures. Alzheimers Dement 8:131-168

2. Ratnavalli E, Brayne C, Dawson K, Hodges JR (2002) The prevalence of frontotemporal dementia. Neurology 58:1615-1621

3. Bohnen NI, Djang DS, Herholz K, Anzai Y, Minoshima S (2011) Effectiveness and safety of ${ }^{18} \mathrm{~F}$-FDG PET in the evaluation of dementia: a review of the recent literature. J Nucl Med 53:59-71

4. Jack CR Jr, Knopman DS, Jagust WJ et al (2013) Tracking pathophysiological processes in Alzheimer's disease: an updated hypothetical model of dynamic biomarkers. Lancet Neurol 12:207-216

5. Hoffman JM, Welsh-Bohmer KA, Hanson M et al (2000) FDG PET imaging in patients with pathologically verified dementia. J Nucl Med 41:1920-1928

6. Minoshima S, Giordani B, Berent S, Frey KA, Foster NL, Kuhl DE (1997) Metabolic reduction in the posterior cingulate cortex in very early Alzheimer's disease. Ann Neurol 42:85-94

7. Mosconi L, Tsui WH, Herholz K et al (2008) Multicenter standardized ${ }^{18} \mathrm{~F}$-FDG PET diagnosis of mild cognitive impairment, Alzheimer's disease, and other dementias. J Nucl Med 49:390-398

8. Jeong Y, Cho SS, Park JM et al (2005) ${ }^{18} \mathrm{~F}$-FDG PET findings in frontotemporal dementia: an SPM analysis of 29 patients. J Nucl Med 46:233-239

9. Diehl-Schmid J, Grimmer T, Drzezga A et al (2007) Decline of cerebral glucose metabolism in frontotemporal dementia: a longitudinal ${ }^{18}$ F-FDG-PET study. Neurobiol Aging 28:42-50

10. Grimmer T, Diehl J, Drzezga A, Forstl H, Kurz A (2004) Regionspecific decline of cerebral glucose metabolism in patients with frontotemporal dementia: a prospective ${ }^{18} \mathrm{~F}$-FDG-PET study. Dement Geriatr Cogn Disord 18:32-36

11. Foster NL, Heidebrink JL, Clark CM et al (2007) FDG-PET improves accuracy in distinguishing frontotemporal dementia and Alzheimer's disease. Brain 130:2616-2635
12. Panegyres PK, Rogers JM, McCarthy M, Campbell A, Wu JS (2009) Fluorodeoxyglucose-positron emission tomography in the differential diagnosis of early-onset dementia: a prospective, communitybased study. BMC Neurol 9:41

13. Rabinovici GD, Rosen HJ, Alkalay A et al (2011) Amyloid vs FDGPET in the differential diagnosis of AD and FTLD. Neurology 77: 2034-2042

14. Roy CS, Sherrington CS (1890) On the regulation of the bloodsupply of the brain. J Physiol 11:85-158

15. Alsop DC, Detre JA (1998) Multisection cerebral blood flow MR imaging with continuous arterial spin labeling. Radiology 208:410 416

16. Detre JA, Leigh JS, Williams DS, Koretsky AP (1992) Perfusion imaging. Magn Reson Med 23:37-45

17. Takahashi H, Ishii K, Hosokawa C et al (2013) Clinical application of 3D arterial spin-labeled brain perfusion imaging for Alzheimer disease: comparison with brain perfusion SPECT. AJNR Am J Neuroradiol

18. Alsop DC, Dai W, Grossman M, Detre JA (2010) Arterial spin labeling blood flow MRI: its role in the early characterization of Alzheimer's disease. J Alzheimers Dis 20:871-880

19. Binnewijzend MA, Kuijer JP, Benedictus MR et al (2012) Cerebral blood flow measured with $3 \mathrm{D}$ pseudocontinuous arterial spinlabeling MR imaging in Alzheimer disease and mild cognitive impairment: a marker for disease severity. Radiology 267:221-230

20. Dai W, Lopez OL, Carmichael OT, Becker JT, Kuller LH, Gach HM (2009) Mild cognitive impairment and Alzheimer disease: patterns of altered cerebral blood flow at MR imaging. Radiology 250:856-866

21. Johnson NA, Jahng GH, Weiner MW et al (2005) Pattern of cerebral hypoperfusion in Alzheimer disease and mild cognitive impairment measured with arterial spin-labeling MR imaging: initial experience. Radiology 234:851-859

22. Du AT, Jahng GH, Hayasaka S et al (2006) Hypoperfusion in frontotemporal dementia and Alzheimer disease by arterial spin labeling MRI. Neurology 67:1215-1220

23. Hu WT, Wang Z, Lee VM, Trojanowski JQ, Detre JA, Grossman M (2010) Distinct cerebral perfusion patterns in FTLD and AD. Neurology 75:881-888

24. Chen Y, Wolk DA, Reddin JS et al (2011) Voxel-level comparison of arterial spin-labeled perfusion MRI and FDG-PET in Alzheimer disease. Neurology 77:1977-1985

25. Musiek ES, Chen Y, Korczykowski M et al (2012) Direct comparison of fluorodeoxyglucose positron emission tomography and arterial spin labeling magnetic resonance imaging in Alzheimer's disease. Alzheimers Dement 8:51-59

26. van der Flier WM, Pijnenburg YA, Prins N et al (2014) Optimizing patient care and research: the Amsterdam Dementia Cohort. J Alzheimers Dis 41:313-327

27. McKhann GM, Knopman DS, Chertkow H et al (2011) The diagnosis of dementia due to Alzheimer's disease: recommendations from the National Institute on Aging-Alzheimer's Association workgroups on diagnostic guidelines for Alzheimer's disease. Alzheimers Dement 7:263-269

28. Rascovsky K, Hodges JR, Knopman D et al (2011) Sensitivity of revised diagnostic criteria for the behavioural variant of frontotemporal dementia. Brain 134:2456-2477

29. Ossenkoppele R, Zwan MD, Tolboom N et al (2012) Amyloid burden and metabolic function in early-onset Alzheimer's disease: parietal lobe involvement. Brain 135:2115-2125

30. Ossenkoppele R, Tolboom N, Foster-Dingley JC et al (2012) Longitudinal imaging of Alzheimer pathology using [(11)C]PIB, [(18)F]FDDNP and [(18)F]FDG PET. Eur J Nucl Med Mol Imaging

31. Jenkinson M, Smith S (2001) A global optimisation method for robust affine registration of brain images. Med Image Anal 5:143-156 
32. Liu Y, Zhu X, Feinberg D et al (2012) Arterial spin labeling MRI study of age and gender effects on brain perfusion hemodynamics. Magn Reson Med 68:912-922

33. Edison P, Archer HA, Hinz R et al (2007) Amyloid, hypometabolism, and cognition in Alzheimer disease: an [(11)C]PIB and $\left[{ }^{18} \mathrm{~F}\right] \mathrm{FDG}$ PET study. Neurology 68:501-508

34. Koedam EL, Lehmann M, van der Flier WM et al (2011) Visual assessment of posterior atrophy development of a MRI rating scale. Eur Radiol 21:2618-2625

35. Borroni B, Grassi M, Premi E et al (2012) Neuroanatomical correlates of behavioural phenotypes in behavioural variant of frontotemporal dementia. Behav Brain Res 235:124-129

36. Binnewijzend MA, Kuijer JP, van der Flier WM et al (2014) Distinct perfusion patterns in Alzheimer's disease, frontotemporal dementia and dementia with Lewy bodies. Eur Radiol 24:2326-2333
37. Ibanez V, Pietrini P, Alexander GE et al (1998) Regional glucose metabolic abnormalities are not the result of atrophy in Alzheimer's disease. Neurology 50:1585-1593

38. Shimizu S, Zhang Y, Laxamana J et al (2010) Concordance and discordance between brain perfusion and atrophy in frontotemporal dementia. Brain Imaging Behav 4:46-54

39. Meltzer CC, Zubieta JK, Brandt J, Tune LE, Mayberg HS, Frost JJ (1996) Regional hypometabolism in Alzheimer's disease as measured by positron emission tomography after correction for effects of partial volume averaging. Neurology 47:454-461

40. Hutton BF, Thomas BA, Erlandsson K et al (2013) What approach to brain partial volume correction is best for PET/MRI? Nucl Inst Methods Phys Res A 29-33 